\title{
A Study of Lung Epithelial Atypia in Regard to the Effect of Smoking and Traffic-Related Air Pollution in Sudan
}

\author{
Hussain Gadelkarim Ahmed ${ }^{*}$ and Twhida Mahdi Rezgalla ${ }^{2}$ \\ ${ }^{I}$ Department of Histopathology and Cytology, Faculty of Medical Laboratory Sciences, University of Khartoum, Sudan \\ ${ }^{2}$ Faculty of Medical Laboratory Sciences, Sudan University for Science and Technology, Khartoum, Sudan
}

\begin{abstract}
Background: Lung cancer risk in association with smoking was well established, but its association with trafficrelated air pollution still unclear. To determine the relationship between exposure to smoking and air pollutants and lung epithelial atypia, we assessed using cytological method; cytological changes in sputum specimens.

Methods: Sputum specimens were obtained from 300 apparently healthy volunteers, living in the city of Khartoum. Of the 300 study subjects, 150 were Traffic policemen (they were exposed to traffic-related air pollution (ascertained as Cases)) and 150 were non-exposed (ascertained as Controls).

Results: Dysplasia was detected in nine individuals, of whom seven were cases and two were controls. All the nine individuals with dysplasia were smokers. Consequently, the risk of dysplasia associated with smoking was found to be statistically significant $(P<0.02)$. Notably, there were 84 individuals with metaplasia, of whom, 58(69\%) were identified among cases and the remaining $26(31 \%)$ were among controls. As a result, the risk of metaplasia associated with smoking and traffic-related air pollution was found to be statistically significant $(P<0.001)$. In respect to the duration of exposure to traffic-related-air pollution among cases, metaplasia increases with the increasing of exposure $(\mathrm{P}<0001)$.
\end{abstract}

Conclusion: Exposure to traffic was also associated with borderline elevated risks for developing dysplasia and high risks of development of metaplasia. Sputum cytology may provide a useful method in the assessment of lung atypical changes.

Keywords: Lung epithelial, traffic-related, air pollution, Sudan.

\section{INTRODUCTION}

Lung cancer is the most common cause of cancer mortality world wide, causing approximately 1.2 million deaths per year [1] and 1.2 million new cases [2]. The incidence rates of lung cancer may vary in different parts of the world. The highest rates have been reported as occurring in developed countries and some developing countries that experienced to the habit of tobacco smoking [3].

The most common lung cancer risk factors are tobacco smoking and environmental air pollution $[4,5]$. Stronger associations between air pollution and lung cancer in neversmokers have been reported by the American Cancer Society study [6]. Evidence that air pollution is a (moderate) risk factor for lung cancer mortality has also been found in several case-control studies [7, 8]. Strong association between cigarette smoking and lung cancer incidence, have been extensively reported [9-12]. Most studies on long-term exposure to air pollution and lung cancer risk have investigated the association with lung cancer mortality. However, in this, we assessed using cytological method lung epithelial atypia to determine the relationship between exposure to smoking and Traffic-related air pollution and lung epithelial atypia in Sudan, and this is the first study in this context from the Sudan.

*Address correspondence to this author at the University of Khartoum, 102, Faculty of Medical Laboratory Sciences, Khartoum, Sudan: E-mail: Hussaingad1972@yahoo.com

\section{MATERIALS AND METHODS}

\section{Study Design}

The Retrospective Cohort has been described in detail [13] (Ahmed et al. 2003). Briefly, this Sudanese Retrospective Cohort Study on traffic-related air pollution was initiated in October 2008 with the enrollment of 300 apparently healthy volunteers (150 policemen "exposed" and 150 indoors employees "non-exposed") aged 20 to 64 years living in the city of Khartoum, Sudan. The study was designed as a case/control-cohort study, ie, cases were derived from the entire policemen who exposed to trafficrelated air pollution for at least 8 hours per day, whereas the person-years at risk were estimated from a random subcohort $(\mathrm{N}=5000)$; controls were derived from individuals working in closed offices for at least 8 hours per day. This approach was chosen for efficient processing of the baseline questionnaire. At baseline, all participants completed a purposeful structured questionnaire on dietary habits and other potential risk factors for cancer. For all participants, information about sex and age at baseline was known (with information about smoking status). Approximately $99 \%$ of policemen in the Sudan are men, and therefore, all study subjects in this study were men.

Details of the exposure assessment have been described according to long-term exposure to outdoor air pollution at the main streets in the city of Khartoum. Ninety percent of participants had lived for the last 10 years or longer in the city of Khartoum. We used traffic intensity of a road with 
traffic intensity $>10,000$ motor vehicles per 24 hours (motor vehicles/24 hours). Seventy five (50\%) of cases or controls were cigarette smokers. Those with family history of lung cancer or with other cancers were excluded from both cases and controls.

\section{Sample Size}

The sample size was calculated using software known as the survey system, available at http: /www. surveystem.com. sscalc.htm. The system inertly relies on the equation: $\mathrm{n}=$ $\mathrm{z} 2 \mathrm{pq} / \mathrm{d} 2$ ( $\mathrm{n}=$ sample size; $\mathrm{z}=$ the standard normal deviate, usually set at 1.96, which corresponds to the level of the $95 \%$ confidence level; $\mathrm{p}=$ the proportion to the target population i.e. percentage of the studied group, which is 0.11 in this study; $q=1.0-p$ ). The case subjects were selected from the police men who worked in Khartoum city during the period from June 2008 to July 2009, the sample size was calculated from the total number of the policemen in the city of Khartoum. On the other hand, the sample size for control was set as 150, without referring to a specific equation for calculation, and they represented the apparently healthy indoors employees.

\section{Specimen Collection}

Sputum specimens were taken form each participant for three consecutive days. For the collection of the sputum specimen, each study subject was given sputum container, and asked to provide early morning expectorate (by deep cough) before food intake or tooth paste use and to take it to the Laboratory as soon as possible. Specimens were prepared within a class 1 biological safety cabinet, the specimen was decanted in to a Petri-dish, and the purulent area was selected to prepare the smear on cleaned micro-slide. The smear was fixed immediately in $95 \%$ ethyl alcohol while it was wet.

\section{Specimen Processing}

The smears were stained using the Papanicolaou staining method. Ethyl alcohol fixed smears were hydrated in descending concentrations of $95 \%$ alcohol through $70 \%$ alcohol to distilled water, for two minutes in each stage. Then the smears were treated with Harris' hematoxylin for five minutes to stain the nuclei, rinsed in distilled water and differentiated in $0.5 \%$ aqueous Hydrochloric Acid for a few seconds, to remove the excess stain. They were then immediately rinsed in distilled water, to stop the action of discoloration. Then the smears were blued in alkaline water for a few seconds and dehydrated in ascending alcoholic concentrations from $70 \%$, through two changes of $95 \%$ alcohol for two minutes for each change. The smears were next treated with Eosin Azure 50 for four minutes. For cytoplasmic staining, they were treated with Papanicolaou Orange G6 for two minutes, rinsed in $95 \%$ alcohol and then dehydrated in absolute alcohol. The smears were then cleared in Xylene and mounted in DPX (Distrene Polystyrene Xylene) mount. All the reagents used were from Thermo Electron Corporation, UK.

\section{Assessment of the Results}

To increase the reliability and reproducibility, strict quality control measures were applied. We included 10 smears from patients with histopathologically diagnosed lung cancer, dysplasia and metaplasia to serve as positive control. In assessing the quality of staining, the smears were examined under low (10X) power using a light microscope. All included smears showed satisfactory staining quality. To avoid the assessment bias, cytological smears were labeled in such a way that the examiner was blinded to the group (case or control) of each subject.

\section{Statistical Analysis}

Traffic-related air pollution effects were analyzed for overall exposure to pollutants and for a combination of cigarette smoking of the pollutants and traffic variables to identify effects on the epithelial cells of the lung. Relative risks (RRs) and 95\% confidence intervals (95\% CIs) were calculated. Further, we conducted subgroup analyses for cigarette smoking status (current). We conducted sensitivity analyses for cigarette smoke and the traffic variables to evaluate the Potential confounding effect of the number of cigarettes, and the number of years of smoking.

Data management was done using Statistical Package for Social Sciences (SPSS version 12). SPSS was used for analysis and to perform Pearson Chi-square test for statistical significance ( $P$ value). The 95\% confidence level and confidence intervals were used.

\section{Ethical Consent}

The study was approved by Faculty of Medical Laboratory Science boards from Sudan University for Science and Technology. All study subjects consented to participation by completing the self-administered questionnaire.

\section{RESULTS}

Description of the cases and the controls by demographic characteristics is shown in Table $\mathbf{1}$. The age distribution was relatively similar among the cases and the controls. The vast majority of the cases and the controls belonged to the 26-35 years' age range and constituted $29.3 \%$ and $21 \%$ of the cases and the controls respectively.

There was a considerable contrast in air pollution exposure among the full cohort (Table 1). Distributions for the traffic variables were skewed regardless to smoking.

Dysplasia was detected in nine $(3 \%)$ individuals, of whom seven $(2.3 \%)$ were cases and two $(0.7 \%)$ were controls. All the nine individuals with dysplasia were cigarette smokers, as shown in Table $\mathbf{2}$ (Fig. 1). Consequently, the risk of dysplasia associated cigarette smoking was found to be statistically significant, by Chisquare test $(\mathrm{P}<0.01)$. Notably, there were $84 \quad(28 \%)$ individuals with metaplasia, of whom, $63(21 \%)$ were identified with few cells showing metaplasia and $21(7 \%)$ with numerous cells showing metaplasia. Of the $63(100 \%)$ smears showing few metaplastic cells, $44(70 \%)$ were detected among cases and the remaining 19(30\%) were found among controls. Out of the 21 (100\%) showing numerous metaplastic cells, 14 (74\%) were among cases and the remaining $7(26 \%)$ were among controls. As a result, the risk of metaplasia associated with smoking and trafficrelated air pollution was found to be statistically significant, by Chi-square test $(P<0.0001)$, as indicated in Tables $\mathbf{1}$ and $\mathbf{3}$ (Fig. 1). 
Table 1. Distribution of Cases and Controls by Age, Dysplasia and Metaplasia

\begin{tabular}{|l|c|c|c|c|c|c|}
\hline \multirow{2}{*}{ Age } & \multicolumn{4}{|c|}{ Studied Subjects } & \multicolumn{2}{c|}{ Total } \\
\cline { 2 - 5 } & \multicolumn{2}{|c}{ Cases } & \multicolumn{2}{c|}{ Controls } & \multicolumn{2}{c|}{} \\
\cline { 2 - 5 } & No & $\%$ & No & $\%$ & No & $\%$ \\
\hline \hline$<25$ years & 23 & 15.3 & 33 & 22 & 56 & 18.7 \\
\hline $26-35$ & 88 & 58.7 & 63 & 42 & 151 & 50.3 \\
\hline $36-45$ & 31 & 20.7 & 41 & 27.3 & 72 & 24 \\
\hline $46^{+}$ & 8 & 5.3 & 13 & 8.7 & 21 & 7 \\
\hline Total & 150 & 100 & 150 & 100 & 300 & 100 \\
\hline Dysplasia & No & $\%$ & No & $\%$ & No & $\%$ \\
\hline Present & 7 & 4.7 & 2 & 1.3 & 9 & 3 \\
\hline Absent & 143 & 95.3 & 148 & 98.7 & 291 & 97 \\
\hline Total & 150 & 100 & 150 & 100 & 300 & 100 \\
\hline Metaplasia & No & $\%$ & No & $\%$ & No & $\%$ \\
\hline present & 58 & 38.7 & 26 & 17.3 & 84 & 28 \\
\hline absent & 92 & 61.3 & 124 & 82.7 & 216 & 72 \\
\hline Total & 150 & 100 & 150 & 100 & 300 & 100 \\
\hline
\end{tabular}

Table 2. Destribution of Study Population by Smoking and Dysplastic Changes

\begin{tabular}{|c|c|c|c|c|c|}
\hline \multirow{3}{*}{ Sample } & \multirow{2}{*}{} & & \multicolumn{2}{|c|}{ Dysplastic } & \multirow{2}{*}{ Total } \\
\cline { 3 - 6 } & & & Present & Absent & \\
\hline \hline \multirow{3}{*}{ Test } & smoking & yes & 7 & 68 & 75 \\
\cline { 2 - 6 } & & No & 0 & 75 & 75 \\
\cline { 2 - 6 } & Total & & 7 & 143 & 150 \\
\hline \multirow{3}{*}{ Control } & smoking & yes & 2 & 73 & 75 \\
\cline { 2 - 6 } & & No & 0 & 75 & 75 \\
\cline { 2 - 6 } & Total & & 2 & 148 & 150 \\
\hline
\end{tabular}

Table 3. Destribution of Study Population by Smoking and Metaplasia

\begin{tabular}{|c|c|c|c|c|c|c|}
\hline \multirow{2}{*}{ Sample } & & & \multicolumn{3}{|c|}{ Metaplastic } & \multirow{2}{*}{ Total } \\
\hline & & & Few & Neumras & Non & \\
\hline \multirow[t]{3}{*}{ Test } & smoking & yes & 34 & 12 & 29 & 75 \\
\hline & & no & 10 & 2 & 63 & 75 \\
\hline & \multicolumn{2}{|c|}{ Total } & 44 & 14 & 92 & 150 \\
\hline \multirow[t]{3}{*}{ Control } & smoking & yes & 11 & 4 & 60 & 75 \\
\hline & & no & 8 & 3 & 64 & 75 \\
\hline & \multicolumn{2}{|c|}{ Total } & 19 & 7 & 124 & 150 \\
\hline
\end{tabular}

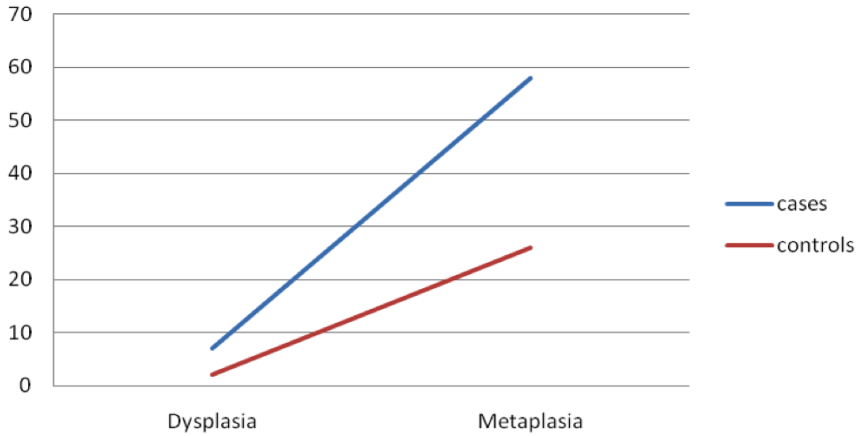

Fig. (1). Description of the study population by lung epithelial atypical changes.

In respect to the duration of exposure to traffic-related air pollution among cases, in duration 21+ years, 13 persons were found with metaplasia representing $87 \%$ of the individuals in this group, followed by 16-20 years, in which metaplasia was demonstrated in 9 cases, representing $69 \%$ of individuals in this group. In duration $11-15$ years, 15 persons were found with metaplasia representing $44 \%$ of the individuals in this group, followed by 6-10 years, in which metaplasia was demonstrated in 16 cases, representing $30 \%$ of individuals in this group. In duration $<5$ years, 5 persons were found with metaplasia representing $14 \%$ of the individuals in this group. Metaplasia increases with the increasing of exposure and this was found to be statistically significant $\mathrm{P}<0001$.

When correlating the relation between metaplasia and smoking among smokers in both groups (cases and controls), $46(30.7 \%)$ of the cases were found with metaplasia, hence, only $15(10 \%)$ of the controls were found with metaplasia, as indicated in Fig. (2). Smoking is risk factor that increases the risk of occurrence of metaplasia that resulting from traffic pollution and this was found to be statistically significant $\mathrm{P}<001$.

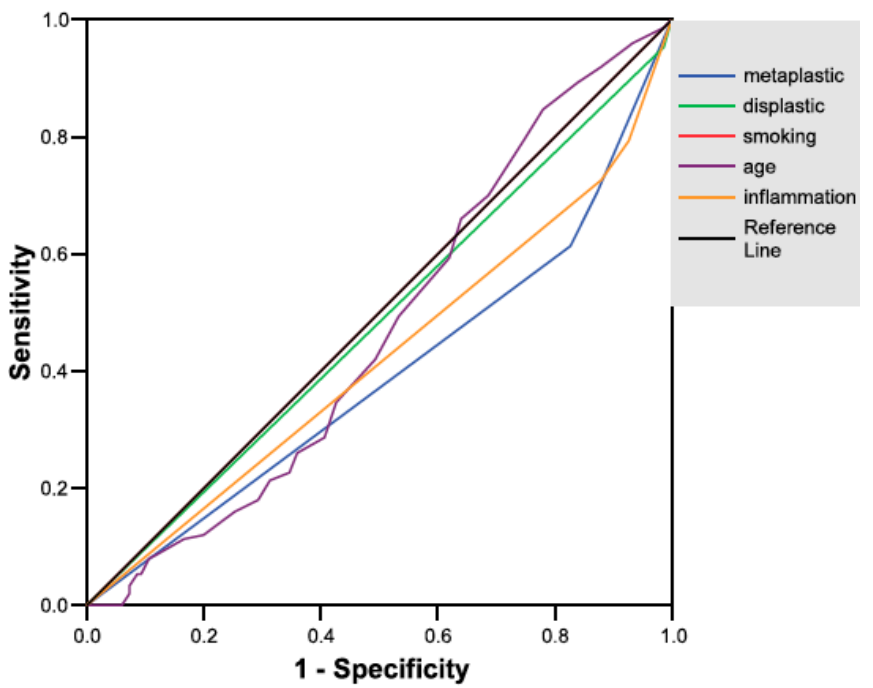

Fig. (2). Description of the study population by different population.

In regard to the relationship between inflammatory infiltrate and exposure, 59 (19.7\%) persons were identified 
with inflammatory infiltrate. Of the $59(100 \%)$ inflammatory infiltrate demonstrated in the whole study population, 41(69\%) were detected among cases and 18(31\%) among controls. Accordingly, the risk of inflammatory infiltrate associated with smoking and traffic-related air pollution was found to be statistically significant, by Chi-square test $(P<0.001)$.

In reference to the relationship between age and metaplasia, high frequencies of metaplasia were found among age groups, 36-45years followed by $46+, 26-35$ and $<$ 25 , constituting, $44 \%, 43 \%, 26 \%$ and $7 \%$ respectively in respect to the total number of individuals in each age group, as indicated in Fig. (2).

\section{DISCUSSION}

To assess the long-term impact of environmental air pollution and tobacco smoking on lung cancer risk, this study investigated by cytological methods two distinct occupational groups. The first group was policemen (exposed for at least 8 hours per day for traffic-related air pollution (cases group)), who were further sub-grouped in to smokers and non-smokers. The second group comprised of individuals working in closed offices for at least 8 hours per day (non-exposed or control group), who were further subgrouped in to smokers and non-smokers.

In this context, we found no association of traffic-related air pollution with lung dysplasia, among people who had never smoked cigarettes. There were positive associations of cigarette smoke and traffic variables with lung epithelial dysplasia.

Stronger associations between traffic-related air pollution and lung metaplasia have been observed in cases, as well as, the relative risk increases with cigarette smoking.

Dysplasia which is believed to be precursors of squamous cell carcinoma [14] was demonstrated in seven $(78 \%)$ of the cases and $2(22 \%)$ of the controls $(\mathrm{RR}=3.5 \%)$. Not surprisingly, smoking status had a huge impact on lung cancer risk. The relative risk for lung cancer ranged from $=$ 4.58 in former smokers to 36.47 in heavy smokers (the comparison was with those who had never smoked) [15]. Cigarette smoking is the major cause for lung cancer, but genetic factors also affect susceptibility. Amos et al. [16] studied families that included multiple relatives affected by lung cancer. Results from linkage analysis showed strong evidence that a region of chromosome $6 \mathrm{q}$ affects lung cancer risk. Unexpectedly few data have been gathered on the long-term health effects of air pollution, including its influence on the development of lung cancer. Most studies on the association between lung cancer and air pollution have investigated mortality [17]. To determine what, if any, additive risk was presented by ambient air pollution, we focused on Policemen, as they are continuously exposed to the highest concentrations of air pollutants with prolonged durations. Motor vehicles and other mobile air pollution sources, such as trucks, trains, buses and factories have been linked to the cancer risk [18]. Although, all cases of dysplasia in this study were smokers $(7$ cases and 2 controls), but the frequency was increased among cases. Although, variation was found to be statistically insignificant, but it might elevate the predictability that, the combined exposure to tobacco smoking and air pollution increases the risk of lung dysplasia. Stronger associations between air pollution and lung cancer in never-smokers have been observed in the American Cancer Society study [6]. In a study covered 15 million people aged 30-64 years in the 1960, 1970, 1980/1981 and/or 1990 censuses in Denmark, Finland, Iceland, Norway and Sweden, and the 2.8 million incident cancer cases diagnosed in these people in a followup until about 2005; Male waiters and tobacco workers had the highest risk of lung cancer, probably attributable to active and passive smoking. Miners and quarry workers also had a high risk, which might be related to their exposure to silica dust and radon daughters. Among women, tobacco workers and engine operators had a more than fourfold risk as compared with the lung cancer risk among farmers, gardeners and teachers. The occupational risk patterns were quite similar in all main histological subtypes of lung cancer [19].

A few studies have investigated the association between air pollution and lung cancer incidence in study populations consisting entirely of nonsmokers. A cohort study in the United States among 6338 nonsmoking adults found that incident lung cancer was elevated with increased air pollutants concentrations. A European case-control study among nonsmokers, have shown that lung cancer incidence was possibly increased with residence nearby heavy traffic roads [20]. Some European studies investigated the association between air pollution exposure and lung cancer incidence using individual-level exposure assessments, with a focus on traffic-related air pollution. These studies suggested an increased risk of lung cancer from trafficrelated air pollution, in particular, assessed by pollutants concentrations or indicator variables for living near a major $\operatorname{road}[15,21]$.

Furthermore, approximately, $86 \%$ of those with dysplasia have been exposed to air pollutants for durations of more than 16 years. Previous studies showed that the association between lung cancer and air pollution became weaker when more recent exposures were used in the analyses, and that exposures 20 years before the start of the study or even earlier in life were most important [15, 22]. A limitation of our study is that we estimated exposure to air pollution based on the period on which the policeman was employed and we used traffic intensity which was not correlated in different years. The intensity of air pollution was only elevated in the recent 10 years in Khartoum city. It was therefore not possible to evaluate the independent contributions of concentrations of different time periods.

Metaplasia is the reversible replacement of one differentiated cell type with another mature differentiated cell type, which is generally caused by some sort of abnormal stimulus. If the stimulus that caused metaplasia is removed or ceases, tissues return to their normal pattern of differentiation. Metaplasia is not synonymous with dysplasia and is not directly considered premalignant but it can develop in to premalignant [10]. In the current study, among cases, air pollution appeared to have a stronger effect particularly on smokers than on controls; and this difference was found to statistically significance $P<0.001$. Most of those with metaplasia among control group were smokers, and role of smoking in inducing of lung metaplasia was well established [23]. Also exposure to different air pollutants 
was linked to epithelium metaplasia in the respiratory tract [24]. Diesel exhaust particles prevented serum starvation-led decreases in epithelial cells by inducing cell cycle progression and preventing apoptosis, processes involving oxidative stress, inhibition of expression and stimulation of $\mathrm{N}$-terminal kinase and nuclear factor-B. Therefore, low-dose diesel exhaust particle exposure may lead to lung epithelial cell hyperplasia. With the increasing use of diesel-powered engines, particulate air pollution is increasingly being recognized as a major public health hazard and as a contributor to the burden of lung diseases. Thus, there is a strong association between particulate air pollution and impaired lung function. In addition, a relationship between mortality and lung metaplasia in adults living in metropolitan areas and the level of particulate air pollution has been reported $[10,25]$. The mechanisms underlying the deleterious effects of particulates on the lung are unclear. Exposure to air pollutants may induce hyperplasia of a normal epithelium by preventing cell apoptosis, perhaps forming the basis for a metaplastic epithelium.

Furthermore, of the 59 inflammatory infiltrate demonstrated in the whole studied subjects, 41(69\%) were detected among cases and 18(31\%) among controls. On exposure to air pollutants and smoking, airway epithelial cells, which form the first line of innate immune defense against particles, produce inflammatory response [25]. In addition, air pollutants and smoking can also cause cell death by necrosis, and by the process of apoptosis, which can increase inflammatory potency $[26,27]$. Some studies have reported that exposure to some air pollutants potentiates acute inflammation and mucus production and secretion elicited by a biogenic substance in rat pulmonary airways [28].

Additionally, most of the cases of dysplasia and metaplasia were detected among elder age groups. The agespecific mortality rates for lung cancer among those living in urban were much higher than younger [29].

In conclusion, there is an association between exposure to both environmental air pollution and tobacco smoking and lung dysplasia. Exposure to traffic was also associated with borderline elevated risks for developing dysplasia and high risks of development of metaplasia.

\section{ACKNOWLEDGEMENT}

The authors are indebted to the participants of this study and further thank people at the General administration for traffic police, for their help and cooperation.

\section{FINANCIAL DISCLOSURE}

The authors have no any connection with any company and the work was supported by university of Khartoum and Sudanese Cancer Society

\section{REFERENCES}

[1] Parking D, Bray F, Ferlay J, et al. Global cancer statistics 2002. CA Cancer J Clin 2005; 55: 74-108.

[2] WHO. Global cancer rates could increase by $50 \%$ to 15 million by 2020, World Cancer Report 2009. Available from: www.who.int/ mediacentre

[3] Jemal A, Siegel R, ward E, et al. Cancer statistics 2008. CA Cancer J Clin 2008; 58: 71-96.

[4] Gustavsson P, Jakobsson R, Nyberg F, et al. Occupational exposure and lung cancer risk: a population-based case-referent study in Sweden. Am J Epidemiol 2009; 152(1): 32-40.
[5] U.S. Department of Health and Human Services, Centers for Disease Control and Prevention, National Center for Chronic Disease Prevention and Health Promotion, Office on Smoking and Health available from: www.cdc.gov/tobacco/dataVistatistics/sgr/s grV̈2004/; 2004.

[6] Pope CA III, Burnett RT, Thun MJ, et al. Lung cancer, cardiopulmonary mortality, and long-term exposure to fine particulate air pollution. JAMA 2002; 287: 1132-41.

[7] Barbone F, Bovenzi M, Cavallieri F, Stanta G. Air pollution and lung cancer in Trieste, Italy. Am J Epidemiol 1995; 141(12): 116169.

[8] Jedrychowski W, Becher $\mathrm{H}$, Wahrendorf J, et al. A case-control study of lung cancer with special reference to the effect of air pollution in Poland. J Epidemiol Commun Health 1990; 44(2): 11420 .

[9] Beelen R, Hoek G, van den Brandt PA, et al. Long-term exposure to traffic-related air pollution and lung cancer risk. Epidemiology 2008; 19(5): 702-10.

[10] Lee JJ, Liu D, Lee JS, et al. Long-term impact of smoking on lung epithelial proliferation in current and former smokers. J Natl Cancer Inst 2001; 93(14): 1081-8.

[11] Peto R, Darby S, Deo H, et al. Smoking, smoking cessation, and lung cancer in the UK since 1950: combination of national statistics with two case-control studies. BMJ 2000; 321(7257): 323-9.

[12] Lubin JH, Blot WJ. Cancer and smoking cessation: patterns of risk. J Natl Cancer Inst 1993; 85: 422-3.

[13] Ahmed HG, Idris AM, Ibrahim SO. Study of oral epithelial atypia among Sudanese tobacco users by exfoliative cytology. Anticancer Res 2003; 23: 1943-9.

[14] Banerjee AK, Rabbitts PH, George PJ. Are all high-grade preinvasive lesions premalignant, and should they all be treated? Am J Respir Crit Care Med 2002; 165: 1452-3.

[15] Nafstad P, Haheim LL, Oftedal B, et al. Lung cancer and air pollution: a 27 year follow up of 16,209 Norwegian men. Thorax 2003; 58: 1071-76.

[16] Amos CI, Pinney SM, Li Y, et al. A susceptibility locus on chromosome 6q greatly increases lung cancer risk among light and never smokers. Cancer Res 2010; 70(6): 2359-67.

[17] Laden F, Schwartz J, Speizer FE, et al. Reduction in fine particulate air pollution and mortality: extended follow-up of the Harvard six cities study. Am J Respir Crit Care Med 2006; 173: $667-2$.

[18] Vineis P, Hoek G, Krzyzanowski M, et al. Air pollution and risk of lung cancer in a prospective study in Europe. Int J Cancer 2006; 119: $169-74$

[19] Pukkala E, Martinsen JI, Lynge E, et al. Occupation and cancer follow-up of 15 million people in five Nordic countries. Acta Oncol 2009; 48(5): 646-790.

[20] Beeson WL, Abbey DE, Knutsen SF. Long-term concentrations of ambient air pollutants and incident lung cancer in California adults: results from the AHSMOG study. Adventist Health Study on Smog. Environ Health Perspect 1998; 106(12): 813-22.

[21] Cho KS, Inoue S, Shinozaki R. Air pollution, temperature, and regional differences in lung cancer mortality in Japan. Arch Environ Health 1997; 52: 160-8.

[22] Nyberg F, Gustavsson P, Jarup L, et al. Urban air pollution and lung cancer in Stockholm. Epidemiology 2000; 11: 487-95.

[23] Lippman JS, Benner SE, Lee JJ, et al. Randomized placebocontrolled trial of isotretinoin in chemoprevention of bronchial squamous metaplasia. J Clin Oncol 1994; 12: 937-45.

[24] Hotchkiss JA, Harkema JR. Effect of neutrophil depletion on endotoxin-induced mucous cell metaplasia in pulmonary airways of F344 rats. Am J Respir Crit Care Med 1994; 149: A994(Abstract).

[25] Bayram HK, Ito $K$, Issa $R$, Ito $M$, Sukkar $M$, Chung KF. Regulation of human lung epithelial cell numbers by diesel exhaust particles. Eur Respir J 2006; 27: 705-13.

[26] Li N, Wang M, Oberley TD, Sempf JM, Nel AE. Comparison of the pro-oxidative and proinflammatory effects of organic diesel exhaust particle chemicals in bronchial epithelial cells and macrophages. J Immunol 2002, 169: 4531-41.

[27] Alfaro-Moreno E, Martinez L, Garcia-Cuellar C, et al. Biologic effects induced in vitro by PM10 from three different zones of Mexico City. Environ Health Perspect 2002; 110(7): 715-20.

[28] Wagner GJ, Van Dyken SJ, Hotchkiss AJ, Harkema JR. Ozone exposure enhances endotoxin-induced mucous cell metaplasia in rat pulmonary airways. Toxicol Sci 2003; 74: 437-46. 
[29] Jie D, Yutang G, Zhongxian W, et al. Cohort study on association of smoking and air pollution with lung cancer among 210,000

persons in shanghai. Chin J Cancer Res 1994; 6(3): 169-72.

Received: March 11, 2010

Revised: March 15, 2010

Accepted: March 22, 2010

(C) Ahmed and Rezgalla et al.; Licensee Bentham Open.

This is an open access article licensed under the terms of the Creative Commons Attribution Non-Commercial License (http://creativecommons.org/licenses/by-nc/

3.0/) which permits unrestricted, non-commercial use, distribution and reproduction in any medium, provided the work is properly cited. 